\title{
Ultrasonic Mechanical, Dialectical and Morphology Characterizations of Unsaturated Copolyesters and Terpolyesters
}

\author{
Momen S. A. Abdelaty \\ Polymer Lap, Chemistry Department, Faculty of Science (Assiut), Al-Azhar University, Assiut, Egypt \\ Email: abdelatymomen@yahoo.com
}

How to cite this paper: Abdelaty, M.S.A. (2017) Ultrasonic Mechanical, Dialectical and Morphology Characterizations of Unsaturated Copolyesters and Terpolyesters. Open Journal of Applied Sciences, 7, 691704.

https://doi.org/10.4236/ojapps.2017.712049

Received: November 15, 2017

Accepted: December 19, 2017

Published: December 22, 2017

Copyright $\odot 2017$ by author and Scientific Research Publishing Inc. This work is licensed under the Creative Commons Attribution International License (CC BY 4.0).

http://creativecommons.org/licenses/by/4.0/

\begin{abstract}
In a previous study we focused on the synthesis of new family of unsaturated copolyesters and terpolyesters by interfacial condensation polymerization. Here, in this study as complementary work of physical characterizations, we focused on the morphological features using X-ray and SEM investigation. Further, the mechanical properties of solid polymers were also studied by a new technique echo-pulse using ultrasonic impulses. This technique was used for solid polymers to determine the elastic moduli involving, Young's modulus, shear modulus, bulk modulus and micro-hardness modulus. The statistical relation between different kinds of copolyesters and terpolyesters with Poisson's ratio $(v)$ and micro-hardness were shown. The dielectric constant $(\mathcal{\varepsilon})$ and the electrical resistance $\left(R_{p}\right)$ of the solid copolyesters and terpolyesters including aliphatic, aromatic and azo group in the main chain have been studied at room temperature. Moreover, the relation between frequency and dielectric constant for the selective polymers has been also illustrated.
\end{abstract}

\section{Keywords}

Unsaturated Copolyesters, Terpolyesters, Ultrasonic Mechanical, Dielectric, Morphology

\section{Introduction}

Because unsaturated polyesters are so widely used, a number of strategies have been suggested [1] [2]. One recent method [3] involves alcoholises of waste resin with propylene glycol, followed by re-esterification with additional maleic anhydride. More styrene is then added to the reconstituted polymer for cross-linking.

Synthesis of polyesters based on biocatalysts and green monomers from re- 
newable resources have extensively studied, due to the eco-friendly product, since both monomers and catalysts are from renewable resources; moreover, polymers are biodegradable [4]-[14]. Nowadays, many studies were focused on the formation of unsaturated polyester composites and their mechanical properties [15] [16]. On the other hand, others studied the unsaturated polyesters as layered silicate nanocomposites [17].

The dielectric constant of material $(\dot{\varepsilon})$ can be defined as the ratio of the capacitance of a condenser containing the material to the capacitance under vacuum referred to. The capacitance of a condenser that measures the extent of charges was stored. An article has focused on studying polymer thin films and their electrical properties with special interest to dielectric behavior for their important applications as insulation, isolation and microelectronics [18] [19]. Moreover, some conducting fillers to improve the magnitude of electrical conductivity of insulating polymers materials have been published in recent articles [20] [21]. The electrical conductivity of insulating polymers materials can be enhanced several orders of magnitude by incorporating some conducting filler in it [22] [23] [24]. The dielectric constant was measured for a variety of materials like cellulosic, [25] protein, and synthetic fibers [26].

Polymers are usually described as viscous liquid and elastic solids. An ideal linear elastic solid obeys Hooke's law; i.e. stress is proportional to the rate of change of strain. An ideal viscous liquid obeys Newton law; i.e. stress is proportional to the rate of change of strain [27]. Ultrasonic method and mechanical spectroscopy are non-destructive testing techniques and can be used for the determination of elastic properties of polymers [28]. Poisson's defined the ratio $(v)$ between transverse strain and longitudinal strain in the elastic loading direction [29]. For most polymer materials Poisson's ratio tending to 0.5 . This can be noticed as a shift from elastic to viscoelastic [30]. Nowadays, many studies were focused on the formation of unsaturated polyester composites and their mechanical properties [31].

This study reports on the morphologic, mechanical and electrical properties of new class of unsaturated copolyesters and terpolyesters. They have been synthetized by interfacial condensation polymerization technique. The synthesis, chemical elucidations and some of physical characterizations have been reported previously.

\section{Experimental}

\subsection{Monomers and Polymers Materials}

They have been reported in previous study [1] [2]. The structures of monomers and polymers under investigations were shown in Scheme 1.

\subsection{Instrumentations and Measurements}

\subsubsection{X-Ray Diffraction Analysis}

$\mathrm{X}$-ray diffractographs of the polymer were obtained with Philips X-ray $\mathrm{Pw} 1710$ 


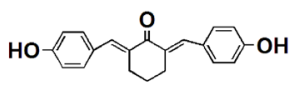

2,6-bis(4-hydroxybenzylidene)cyclohexanone

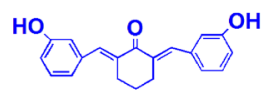

2,6-bis(3-hydroxybenzylidene)cyclohexanone

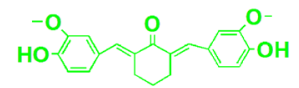

2,6-bis(4-hydroxy-3-methoxybenzylidene)cyclohexanone



I a, b, c
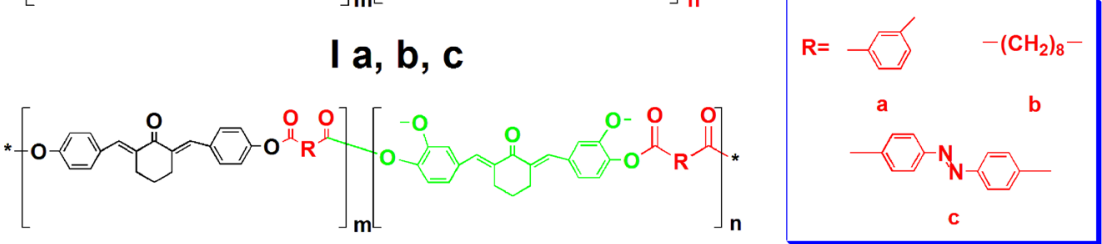

II $a, b, c$

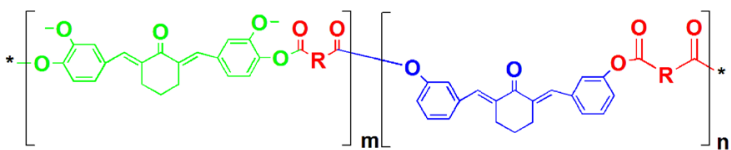

III a, b, c

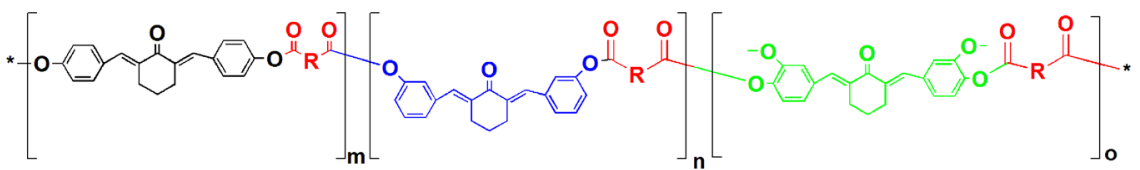

IV a, b, c

Scheme 1. Structures of monomers and polymers have been synthetized and characterized in previous work and are used in the present study.

diffract-meter, using Ni-filtered $\mathrm{CuK} \alpha$ radiation. Please do not revise any of the current designations.

\subsubsection{Ultrasonic Mechanical Characterization Sample Preparation}

\section{1) Formation of Polymer disc}

The solid polymer was grinded by manual mortar. The polymer powder was then taken to QwikHandi-Press (used in IR instrument) under pressure of 5 ton to get a uniform pellet of polymer with a thickness $0.9-1.2 \mathrm{~cm}$.

\section{2) Density measurements}

The densities of polymers were determined at room temperature using the standard displacement method. The polymer sample was hanged by a Teflon wire $(0.01 \mathrm{~mm}$ in diameter) to one arm of sensitive balance. Water was used as immersion liquid. Densities were calculated according to the following equation;

$$
\rho=\rho_{0}\left(W-W_{t}\right) /\left(W-W_{t}\right)\left(W_{1}-W_{1 t}\right)
$$

where $\rho_{0}$ is the density of water $\left(0.9999 \mathrm{~kg} \cdot \mathrm{m}^{-3}\right.$ at RT $\left.25^{\circ} \mathrm{C}\right), W$ and $W_{l}$, are the weight of polymer pellet in air and water respectively, $W_{t}$ and $W_{l t}$ are weight of 
suspended Teflon wire in air and water respectively.

\section{3) Ultrasonic Velocity measurements}

Ultrasonic waves travel through any solid material at a specific velocity related to the material characterization and its density. Here, we used the pulse technique which is widely used to generate ultrasonic measurements for solid and fluids. A pulse of sinusoidal voltage is applied to a piezoelectric transducer that is directly contacted with the samples under investigation. The electrical pulse converted to acoustical pulse that transmitted into the medium. The pulse propagates down the medium, and reflected to the opposite face. The time intervals between successive echoes can be measured and velocity of the wave is determined. Once, time intervals duration is obtained and the thickness of the samples determined the velocity of the ultrasonic wave of the sample can be calculated by Equation (2);

$$
V=2 d / \Delta t
$$

where $d$ is the thickness of the sample and $\Delta t$ is the time intervals (31).

In general, the solids are subjected to three types of stressing condition; uniaxial stress, triaxial stress and pure shear. If a uniaxial stress is applied on a body, then elongation in the same direction will be produced. Young's modulus $(E)$; defined as the ratio of the linear stress to linear strain. Shear of rigidity $(G)$; defined as the ratio of the shear stress to the shear strain. Bulk modulus $(K)$; defined as the ratio of the hydrostatic stress to the volumetric strain. Micro-hardness $(H)$; is the resistant of material to being penetrated and eroded by another material's shear projection. The four constants held as Equations (3)-(7);

$$
\begin{gathered}
L=\rho V_{l}^{2} \\
G=\rho V_{s}^{2} \\
E=2(1+v) G \\
K=L-(4 / 3) G \\
H=(1-2 v) E / 6(1+v)
\end{gathered}
$$

where $\rho$ is the density of the material, $L$ is the longitudinal elastic modulus, $V_{l}$ and $V_{s}$ are the longitudinal and transverse ultrasonic velocities and $v$ is Poisson's ratio [32].

\subsubsection{Electrical Properties}

The dielectric constant $(\hat{\varepsilon})$, parallel resistance $\left(R_{p}\right)$ and parallel capacitance $\left(C_{p}\right)$ were determined for specimens in the form of discs. They were prepared as discussed lately, the diameter $10 \mathrm{~mm}$ and thickness about $7 \mathrm{~mm}$. The measurements have been carried out at room temperature and frequency rang $100 \mathrm{~Hz}-1$ MHz using HIOKI 3532-50 LCR Hi TESTER.

\subsection{Scanning Electronic Microscopy}

The morphology of the polymer were examined by Scanning Electron Micro- 
scopy (SEM) using a JSM-5400 LV instrument; images were recorded with a Pentax Z-50P Camera with Ilford film at an accelerating voltage of $15 \mathrm{KV}$.

\section{Results and Discussions}

\section{1. ${ }^{1} \mathrm{HNMR}$ and IR}

In the previous study we discussed the chemical investigation of these polymers using 1NMR and IR showed good agreement with the chemical structure [1] [2].

\subsection{X-Ray Diffraction}

X-ray diffractograms of selected examples of copolyesters showed an amorphous patterns in the region $2 \theta=5^{\circ}-30^{\circ}$, after this region showed a few reflections of sharpness peaks with an amorphous back ground in the region $2 \theta=30^{\circ}-60^{\circ}$, this indicate some degree of polycrystallinity of the polymer. It was noted from Figure 1 that, the presence of cyclohexyl ring in the polymer chain beside the presence of methylene groups, four or eight, increase the polymer chain flexibility, that urge the approach and mutual attraction of the adjacent chains might be induced some extent of crystallinity. Moreover, it should be noted that the presence of methoxyl groups as substituent in the phenyl ring caused some hindering between the repeating units and enforced it's to the unsymmetrical orientation in the polymer chains and reduced the close packed structure and hence these copolyesters exhibit only a low degree of crystallinity [33]. Moreover, in copolyesters (IVe, $\mathrm{Ve}, \mathrm{Vie}$ ) the presence of $-\mathrm{N}=\mathrm{N}$ - as a polar group in addition to $-\mathrm{C}=\mathrm{C}$ - bonds induces some order between two adjacent chains in the polymer towards some extent of crystallinity [34] as shown in Figure 1. X-ray diffractograms of terpolyesters (IVa, c, d) showed an amorphous halo patterns in the region $2 \theta=5^{\circ}-60^{\circ}$ this indicate a low degree of crystallinity at this region as shown in Figure 1.

Terpolyester (IVb) that contain sebacoyl with eight methylene groups in the

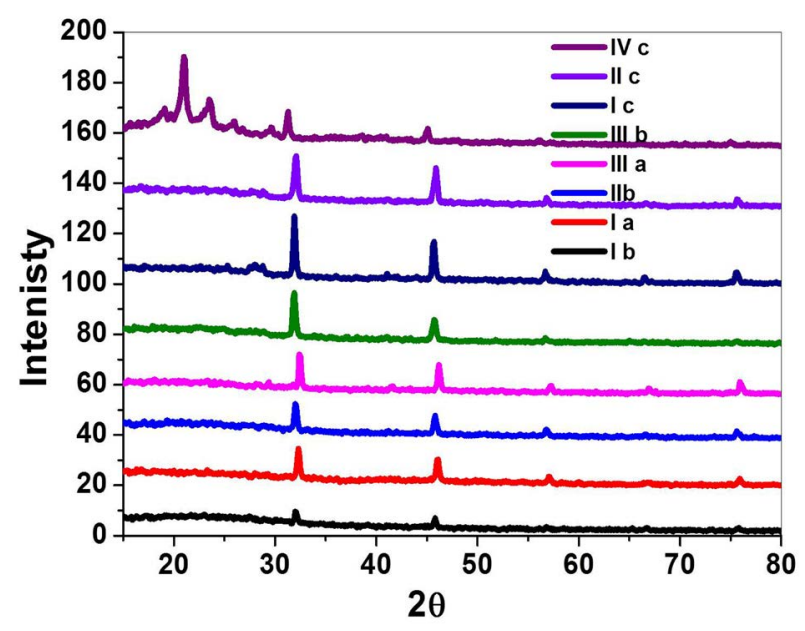

Figure 1. X-ray diffraction of selective copolyesters and terpolyesters. 
main chain is higher crystalline than the other terpolyesters (IVa) due to the approach and mutual attraction of the adjacent chains, which increase the polymer chain flexibility, and induced some extent of crystallinity. Terpolyester (IVc) that contain azo group as a polar group in the main chain have low degree of crystallinity due to the presence of azo group will facilitate the movement of the main chain, therefore it will increase elasticity of polymer [35].

\subsection{Mechanical Properties}

Values of longitudinal, transverse ultrasonic velocities and elastic moduli constants for different copolyesters and terpolyesters based on diarylidenecyclohexanone are cleared in Table 1. The ultrasonic changes may be explained on the basis of physical properties of copolyesters and terpolyesters demonstrated the elastic moduli parameters as shown in Table 2. In polymer (Ib) with aliphatic group (sebacoyl) in the main chain are more elastic than polymer (Ia) with aromatic group (isophthaloyl) in the main chain. This attributed to, the presence of flexible aliphatic units greatly increase the mobility of the copolyesters, and hence have low micro-hardness [35]. The presence of methoxy group in the main chain as in polymer (II a-c), (III a-c) may be increase the intermolecular force of attraction and hinders the three-dimensional packing of the polymer main chain and this copolyesters exhibit low degree of crystallinity [36]. In polymer (Iva-c) terpolyesters, they have higher long chain than copolyesters, elasticity will increase due the effect of the neighbouring group, polymer (IVb) is more elastic than (Iva) as discussed later [37]. Polymer with azo groups in the main chain showed average elasticity between those with aromatic and aliphatic groups, this can be attributed to the mobility of azo group in presence of aromatic chains [35]. The statistical clarifications of different kinds of copolyesters

Table 1. Densities, ultrasonic velocities (longitudinal, transverse) and Poisson's ratio for copolyesters and terpolyesters.

\begin{tabular}{|c|c|c|c|c|}
\hline Polymer & $\rho^{\mathrm{a}}\left(\mathrm{Kg} / \mathrm{m}^{3}\right) 10^{3}$ & $\mathrm{~V}_{\mathrm{L}}^{\mathrm{B}} \quad(\mathrm{m} / \mathrm{s})$ & $\mathrm{V}_{\mathrm{s}}^{\mathrm{c}} \quad(\mathrm{m} / \mathrm{s})$ & $v^{\mathrm{d}}$ \\
\hline Ia & 1.38 & 5855 & 3155 & 0.29 \\
\hline $\mathrm{Ib}$ & 1.22 & 6356 & 3272 & 0.32 \\
\hline Ic & 1.39 & 5945 & 3214 & 0.29 \\
\hline IIa & 1.39 & 5656 & 3327 & 0.23 \\
\hline IIb & 1.28 & 6139 & 3394 & 0.28 \\
\hline IIc & 1.41 & 5657 & 3251 & 0.25 \\
\hline IIIa & 1.37 & 5835 & 3213 & 0.28 \\
\hline IIIb & 1.23 & 6262 & 3285 & 0.31 \\
\hline IIIc & 1.40 & 5900 & 3280 & 0.27 \\
\hline IVa & 1.41 & 6015 & 3234 & 0.29 \\
\hline $\mathrm{IVb}$ & 1.30 & 6527 & 3221 & 0.34 \\
\hline IVc & 1.43 & 6345 & 3245 & 0.32 \\
\hline
\end{tabular}

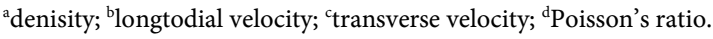


Table 2. Elastic moduli for selected copolyesters and terpolyesters.

\begin{tabular}{cccccc}
\hline Polymer & $(\mathrm{L})^{\mathrm{a}} \mathrm{GPa}$ & $(\mathrm{G})^{\mathrm{b}} \mathrm{GPa}$ & $(\mathrm{E})^{\mathrm{c}} \mathrm{GPa}$ & $(\mathrm{K})^{\mathrm{d}} \mathrm{GPa}$ & $(\mathrm{H})^{\mathrm{e}} \mathrm{GPa}$ \\
\hline Ia & 47.30 & 13.74 & 35.45 & 28.98 & 1.92 \\
Ib & 49.28 & 13.06 & 34.47 & 31.86 & 1.56 \\
Ic & 49.13 & 14.36 & 37.05 & 30.00 & 2.01 \\
IIa & 44.46 & 15.38 & 37.83 & 23.95 & 2.77 \\
IIb & 48.24 & 14.74 & 37.73 & 28.58 & 2.20 \\
IIc & 45.12 & 14.90 & 37.25 & 25.25 & 2.48 \\
IIIa & 46.64 & 14.14 & 36.28 & 27.79 & 2.08 \\
IIIb & 57.57 & 15.06 & 39.75 & 37.50 & 1.81 \\
IIIc & 48.73 & 15.06 & 38.25 & 28.65 & 2.31 \\
IVa & 51.01 & 14.75 & 38.05 & 31.43 & 2.10 \\
IVb & 55.38 & 13.49 & 36.15 & 37.39 & 1.44 \\
IVc & 48.23 & 13.27 & 34.77 & 30.53 & 1.68 \\
\hline
\end{tabular}

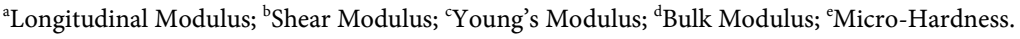

and terpolyesters with Poisson's ratio $(v)$ and micro-hardness $(\mathrm{H})$ were shown Figure 2 and Figure 3 respectively.

\subsection{Electrical Properties}

The dielectric constant $(\hat{\varepsilon})$ and the electrical resistance $R_{p}$ of the two types of copolyesters and terpolyesters including aliphatic, aromatic and azo group in the main chain have been studied carefully at room temperature as clear in Table 3.

Figure 4 showed the relation between frequency and dielectric and these indicating that copolyesters and terpolyesters are good insulator. The difference in the dielectric values can be interpreted to the difference in samples thickness which has a direct relation.

Polymers which contain azo group in main chain show inversely proportional with frequency due to the presence of $(-\mathrm{N}=\mathrm{N}-)$ group with lone pair of electrons on nitrogen atom and $\pi-\pi^{*}, \mathrm{n}-\pi^{*}$ transition which increase polarizability for the samples and decrease with frequency (36). Also the values of the electrical resistance $R_{p}$ of the two groups are about $107 \Omega$. So we can conclude that these copolyesters and terpolyesters can be used as a dielectric medium and as insulator in different applications.

\subsection{Morphological Features (SEM)}

The morphology of the synthesized copolyesters was examined by scanning electron microscopy (SEM). The SEM samples were prepared by putting a smooth part of polymer on a copper holder and subsequently coating it with gold palladium alloy. SEM images were taken on a penta Z50 P Camera with IL ford film at an accelerating voltage of $15 \mathrm{kV}$ using a low-dose technique [37]. 


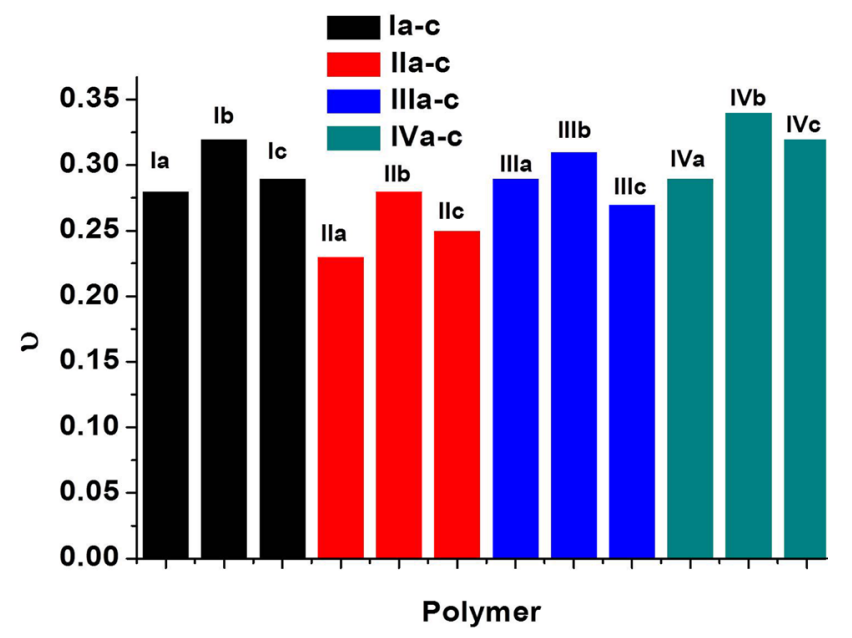

Figure 2. Statistical clarification for copolyesters and terpolyesters with Poisson's ratio $(v)$.

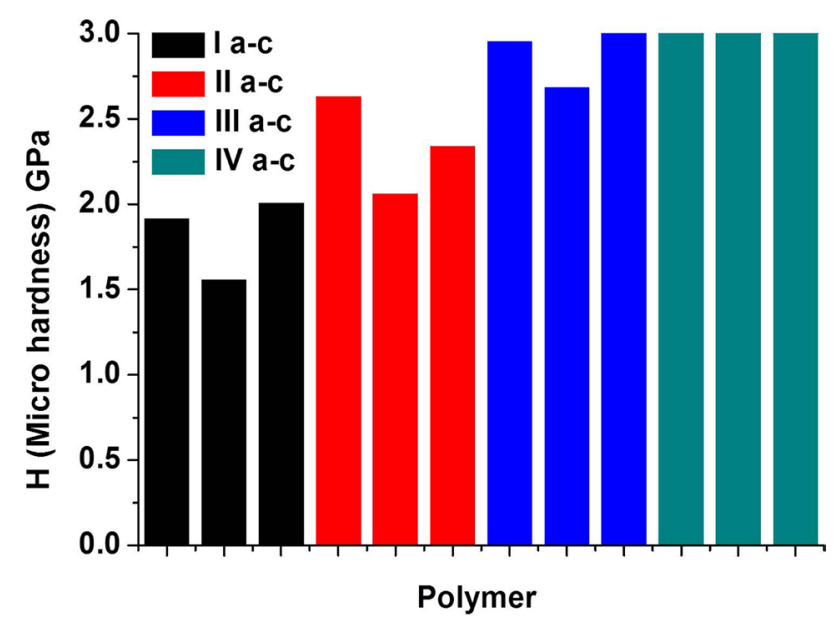

Figure 3. Statistical clarification of copolyesters and terpolyesters with micro-hardness $(\mathrm{H})$.

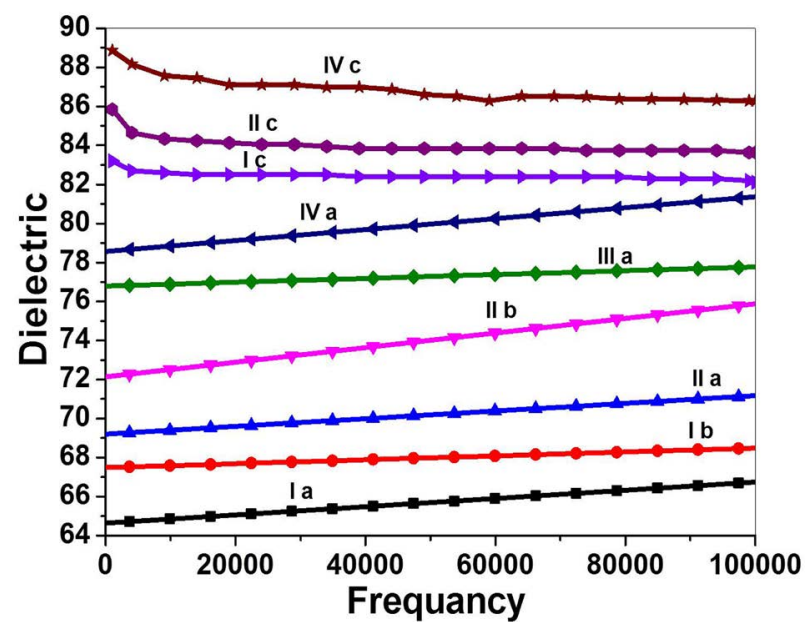

Figure 4. The relation between frequency and dielectric constant for selected copolyesters and terpolyesters. 
Table 3. The values of relative dielectric constant $(\mathcal{\varepsilon})$, parallel resistance $\left(R_{p}\right)$ and parallel capacitance $\left(C_{p}\right)$ for selected copolyesters and terpolyesters.

\begin{tabular}{ccccc}
\hline Polymer & $\mathrm{d}(\mathrm{m}) 10^{-3}$ & $\dot{\varepsilon}^{\mathrm{a}}$ & $\mathbf{R}_{\mathrm{p}}^{\mathrm{b}}(\Omega) 10^{7}$ & $\mathbf{C}_{\mathrm{p}}^{\mathrm{c}} 10^{-11}$ \\
\hline Ia & 2.50 & $62-66$ & $5-8$ & 1.17 \\
Ib & 3.50 & $81-87$ & $5-7$ & 1.09 \\
Ic & 2.50 & $64-65$ & $4-7$ & 1.14 \\
IIa & 3.20 & $80-85$ & $7-9$ & 1.56 \\
IIb & 4.26 & $111-116$ & $6-8.50$ & 1.10 \\
IIc & 1.58 & $43-45$ & $4-6$ & 1.22 \\
IIIa & 1.59 & $41-43$ & $6-8$ & 1.19 \\
IIIb & 4.00 & $98-104$ & $7-9$ & 1.26 \\
IIIc & 5.18 & $132-135$ & $3-6$ & 1.14 \\
IVa & 3.20 & $80-85$ & $7-9$ & 1.55 \\
IVb & 5.18 & $100-110$ & $6-8$ & 1.20 \\
IVc & 1.50 & $70-73$ & $4-6$ & 1.30 \\
\hline
\end{tabular}

a dielectric constant, ${ }^{b}$ parallel resistance, ${ }^{c}$ parallel capacitance.

The study of selected examples of copolyesters showed that the surface of copolyesters (Ia), magnification of $\mathrm{X}=750$ likes scapolite shape which appear as fibrous aggregates. For the surface of copolyesters (Ic), magnification of $\mathrm{X}=750$ likes coral shape. For the surface of copolyesters (IIa), magnification of $\mathrm{X}=350$ appear as granular and compact waxy-looking masses which likes gypstum. For the surface of copolyesters (IIb), magnification of $X=500$, showed granoblastic, mosaic-type, which likes quartzite rock as shown in Figure 5(a). Copolyesters (IIc), magnification of $X=500$ appear as big granular. Figure 5(b) copolyesters (IIIc), magnification of $\mathrm{X}=150$ appear as multi-layer of fibrous particles. Terpolyester (IVa), magnification of $X=200$ likes fibrous aggregates. Figure 5(c) the surface of terpolyesters (IVb), magnification of $X=200$ showed coral shape with porous particles; (IVc), magnification of $X=750$ fibrous aggregates.

\section{Conclusion}

In the present study we completed our work for synthesized copolyesters and terpolyesters which have been discussed in our previous work. The chemical and physical characterization has evaluated. Here we focused our interest in the electrical, mechanical with morphology investigation. The X-ray diffraction showed the amorphous state of polymers with some crystallinity in terpolyesters. Scanning Electron Microscopy (SEM) showed the formation of heterogeneous layers. The dielectric constant of copolyesters and terpolyesters with aliphatic or aromatic chains demonstrated the insulator characters. However, others with azo groups in the main chain demonstrated some electrical properties due to highly polarizability. The mechanical properties of copolyesters and terpolyesters were 
investigated using ultrasonic waves and kinds of elastic moduli have been calculated. The copolyesters and terpolyesters with aliphatic main chain have more elasticity than others with aromatic chains.
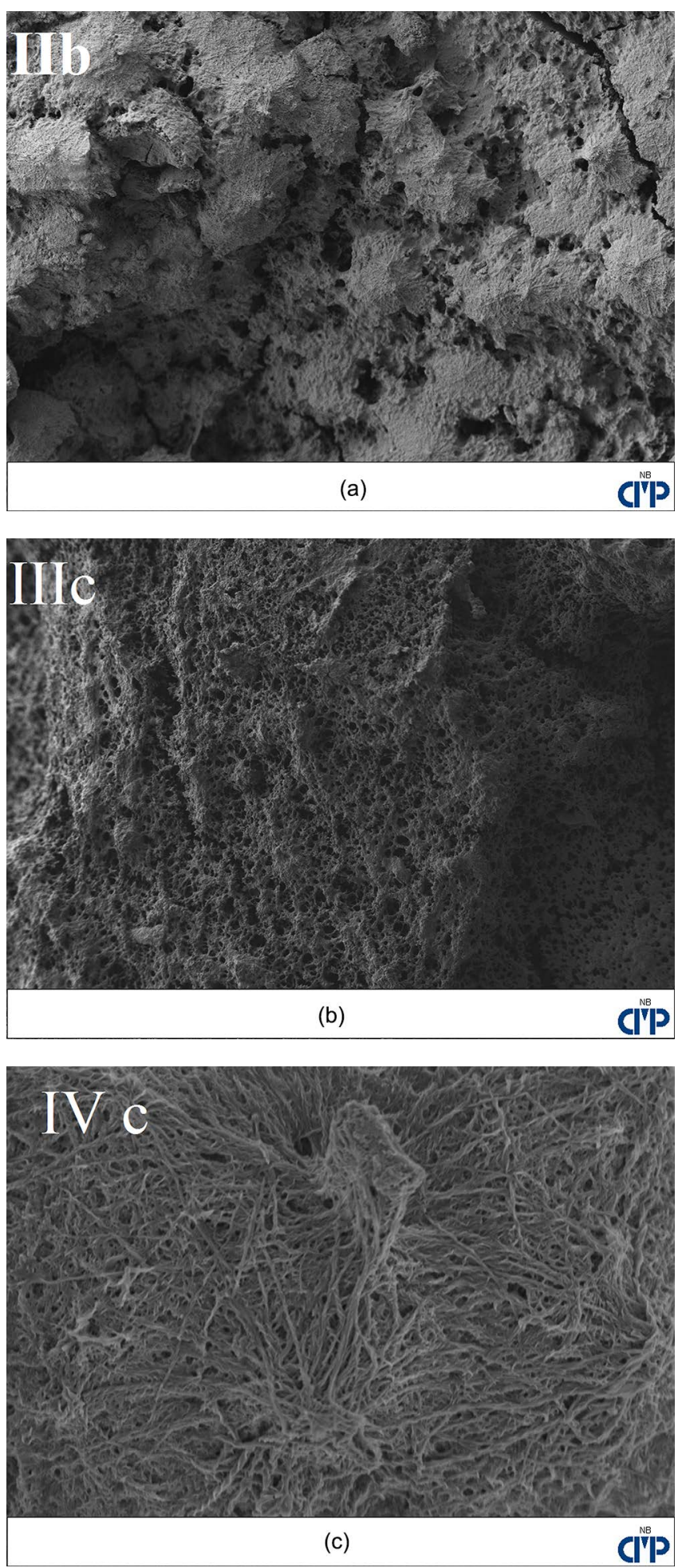

Figure 5. SEM of selected copolyesters and terpolyesters at different magnifications. 


\section{Conflicts of Interest}

The authors declare no conflict of interest.

\section{References}

[1] Abdelaty, M.S.A. (2017) Fabrication and Characterization of Unsaturated Copolyesters Based on Diarylidenecyclohexanone. American Journal of Polymer Science and Technology, 3, 12-20.

[2] Abdelaty, M.S.A. (2017) Fabrication and Characterization of New Unsaturated Terpolyesters Based on Diarylidenecyclohexanone. Polymer Sciences, 3, 1-6. https://doi.org/10.4172/2471-9935.100027

[3] Yoon, K.H., DiBenedetto, A.T. and Huang, S.J. (1997) Recycling of Unsaturated Polyester Resin Using Propylene Glycol. Polymer, 38, 2281-2292.

https://doi.org/10.1016/S0032-3861(96)00951-2

[4] Stavila, E., Alberda van Ekenstein, G.O.R. and Loos, K. (2013) Enzyme-Catalyzed Synthesis of Aliphatic-Aromatic Oligoamides. Biomacromolecules, 14, 1600-1606. https://doi.org/10.1021/bm400243a

[5] Azim, H., Dekhterman, A., Jiang, Z. and Gross, R.A. (2006) Candida Antarctica Lipase B-Catalyzed Synthesis of Poly(butylene succinate): Shorter Chain Building Blocks Also Work. Biomacromolecules, 7, 3093-3097. https://doi.org/10.1021/bm060574h

[6] Jiang, Y. and Loos, K. (2016) Enzymatic Synthesis of Biobased Polyesters. Polymers, 8, 1-53.

[7] Juais, D., Naves, A.F., Li, C., Gross, R.A. and Catalani, L.H. (2010) Isosorbide Polyesters from Enzymatic Catalysis. Macromolecules, 43, 10315-10319.

https://doi.org/10.1021/ma1013176

[8] Ebata, H.K. and Toshima, S. (2008) Lipase-Catalyzed Synthesis and Properties of Poly[(12-hydroxydodecanoate)-co-(12-hydroxystearate)] Directed towards Novel Green and Sustainable Elastomers. Macromolecular Bioscience, 8, 38-45. https://doi.org/10.1002/mabi.200700134

[9] Kobayashi, T. and Matsumura, S. (2012) Enzymatic Synthesis and Chemical Recycling of NovelPolyester-Type Thermoplastic Elastomers. Polymers, 4, 1259-1277. https://doi.org/10.3390/polym4021259

[10] Tsujimoto, T., Uyama, H. and Kobayashi, S. (2001) Enzymatic Synthesis of Cross-Linkable Polyesters from Renewable Resources. Biomacromolecules, 2, 29-31. https://doi.org/10.1021/bm000097h

[11] Tsujimoto, T. and Uyama, H. (2002) Kobayashi Enzymatic Synthesis and Curing of Biodegradable Crosslinkable Polyesters. Macromolecular Bioscience, 2, 329-335. https://doi.org/10.1002/1616-5195(200209)2:7<329::AID-MABI329>3.0.CO;2-H

[12] Uyama, H., Kuwabara, M., Tsujimoto, T. and Kobayashi, S. (2003) Enzymatic Synthesis and Curing of Biodegradable Epoxide-Containing Polyesters from Renewable Resources. Biomacromolecules, 4, 211-215. https://doi.org/10.1021/bm0256092

[13] Jiang, Z., Azim, H., Gross, R.A., Focarete, M.L. and Scandola, M. (2007) Lipase-Catalyzed Copolymerization of $\omega$-Pentadecalactone with $\mathrm{p}$-Dioxanone and Characterization of Copolymer Thermal and Crystalline Properties. Biomacromolecules, 8, 2262-2269. https://doi.org/10.1021/bm070138a

[14] Marques, I.L. (2013) M.Sc University of Coimbra. Development of Unsaturated Polyesters from Renewable Sources. 
[15] Sawpan, M.A., Pickering, K.L. and Fernyhough, A. (2011) Effect of Fibre Treatments on interfacial Shear Strength of Hemp Fibre Reinforced Polylactide and Unsaturated Polyester Composites. Composites Part A: Applied Science and Manufacturing, 42, 1189-1196. https://doi.org/10.1016/j.compositesa.2011.05.003

[16] Bessadok, A., Roudesli, S., Follain, N. and Lebrun, L. (2009) Alfa Fibres for Unsaturated Polyester Composites Reinforcement: Effects of Chemical Treatments on Mechanical and Permeation Properties. Composites Part A, 40, 184-195. https://doi.org/10.1016/j.compositesa.2008.10.018

[17] Motawie, M.A., Ahmed, M., ElMesallamy, S.M., Sadak, E.M. and Kandile, N.G. (2014) Unsaturated Polyesters/Layered Silicate Nanocomposites: Synthesis and Characterization. Journal of Applied Chemistry, 7, 34-43.

[18] Sengwa, R.J. and Sankhla, S. (2008) Characterization of Ionic Conduction and Electrode Polarization Relaxation Oligomers. Polymer Bulletin, 60, 689-700.

https://doi.org/10.1007/s00289-008-0892-Z

[19] Yao, L., He, W., Zuosen, S., Jingjing, M., Xuesong, W., Donghang, Y. and Zhanchen, C. (2015) Novel High-k Polymers as Dielectric Layers for Organic Thin-Film Transistors. Polymer Chemistry, 6, 6651-6658.

https://doi.org/10.1039/C5PY00891C

[20] Shekhar, S., Prasad, V. and Subramanyam, S.V. (2006) Structural and Electrical Properties of Composites of Polymer-Iron Carbide Nanoparticles Embedded in Car Bon. Materials Science and Engineering B, 133, 108-112. https://doi.org/10.1016/j.mseb.2006.06.010

[21] OmedGh, A., Jamal, G.M., Tahir, D.A. and Saeed, S.R. (2011) Electrical Characterization of Polyester Reinforced by Carbon Black Particles. International Journal of Applied Physics and Mathematics, 2, 101-105.

[22] Liu, W., Wang, F., Tan, T. and Chen, B. (2013) Lipase-Catalyzed Synthesis and Characterization of Polymers by Cyclodextrin as Support Architecture. Carbohydrate Polymers, 92, 633-640. https://doi.org/10.1016/j.carbpol.2012.09.064

[23] Ye, C., Ma, G., Fu, W. and Wu, H. (2015) Effect of Fiber Treatment on Thermal Properties and Crystallization of Sisal Fiber Reinforced Polylactide Composites. Journal of Reinforced Plastics and Composites, 34, 718-730. https://doi.org/10.1177/0731684415579090

[24] Bal, K. and KothariIndian, V.K. (2009) Measurement of Dielectric Properties of Textile Materials and Their Applications. Journal of Fibre and Textile Research, 34, 191-199.

[25] Bhowmick, A.K. (1990) Mechanical Properties of Polymers. Materials Science and Engineering, 1.

[26] Samulionis, V., Banys, J., Sanchez-Ferrer, A. and Mezzenga, R. (2015) Ultrasonic and Dielectric Studies of Polyurea Elastomer Composites with Inorganic Nanoparticles. Ferroelectrics, 479, 67-75. https://doi.org/10.1080/00150193.2015.1011979

[27] Greaves, G.N., Greer, A.L., Lakes, R.S. and Rouxel, T. (2011) Poissons Ratio and Modern Material. Nature Materials, 10, 823-837. https://doi.org/10.1038/nmat3134

[28] Papadakis, E.P. (1976) Physical Acoustics Principles and Methods. Academic Press, New York, Vol. 12, 277-374.

[29] Awad, M.S. (2000) Ultrasonic Mechanical of Polyamide. M.Sc. Thesis, Cairo University, Cairo.

[30] Prasad, V.S. and Pillai, K.S. (2001) Synthesis and Thermal Behavior of Thermotropicter Polymers Based on 8-(3-Hydroxyphenyl) Octanoic Acid, 2,6-Naphtha- 
lenedicarboxylic Acid, and Substituted Hydroquinones. Journal of Applied Polymer Science, 82, 1021-1029. https://doi.org/10.1002/app.1936

[31] Madelkern, L. (1964) Crystallization of Polymers. McGraw-Hill, New York.

[32] Abd-Alla, M.A., El-Zohhry, M.F., Aly, K.I. and Mohamed, M.M. (1993) Arylidene Polymers XVIII. Synthesis and Thermal Behavior of Organometallic Arylidene Polymers Containing Ferrocene Derivatives in the Main Chain. Journal of Applied Polymer Science, 47, 323-329. https://doi.org/10.1002/app.1993.070470211

[33] Ebewel, R.O. (1996) Boca Raton, Polymer Science and Technology. Chain Flexability, London, New York, Washington DC, 111.

[34] Gan, Z., Kuwabara, K., Motonri, Y. and Hideki, A. (2004) Solid-State Structures and Thermal Properties of Aliphatic-Aromatic Poly(butylene adipate-co-butylene terephthalate) Copolyesters. Polymer Degradation and Stability, 83, 289-300. https://doi.org/10.1016/S0141-3910(03)00274-X

[35] Ghoneim, L.A., El-Kholy, W.A., Hassan, M.S. and Abadir, M.F. (2011) Characterization of El-Fawakhir Serpentine Fibers and Their Use in the Reinforcement of Unsaturated Polyester. Journal of American Science, 7, 730-736.

[36] Aly, K.I., Hammam, A.S., Radwan, S.M. and Abdel-Rahman, M.A. (2011) New Unsaturated Copolyesters Based on Diarylidenecyclopentanone. Optimum Conditions of Synthesis, Characterization and Morphology. International Journal of Basic \& Applied Sciences IJBAS-IJENS, 11, 14-22.

[37] Tager, A. (1972) Physical Chemistry of Polymers. Mir, Moscow. 


\section{Graphical Abstract}

The present study is accomplishment of our recent work for the synthesis of unsaturated co-and terpolyesters. The aim here is to investigate the electrical and mechanical properties. The dielectric constant of copolyesters and terpolyesters with aliphatic or aromatic chains demonstrated the insulator characters .The mechanical properties of copolyesters and terpolyesters were investigated using ultrasonic waves and kinds of elastic moduli have been calculated. The copolyesters and terpolyesters with aliphatic main chain have more elasticity than others with aromatic chains.

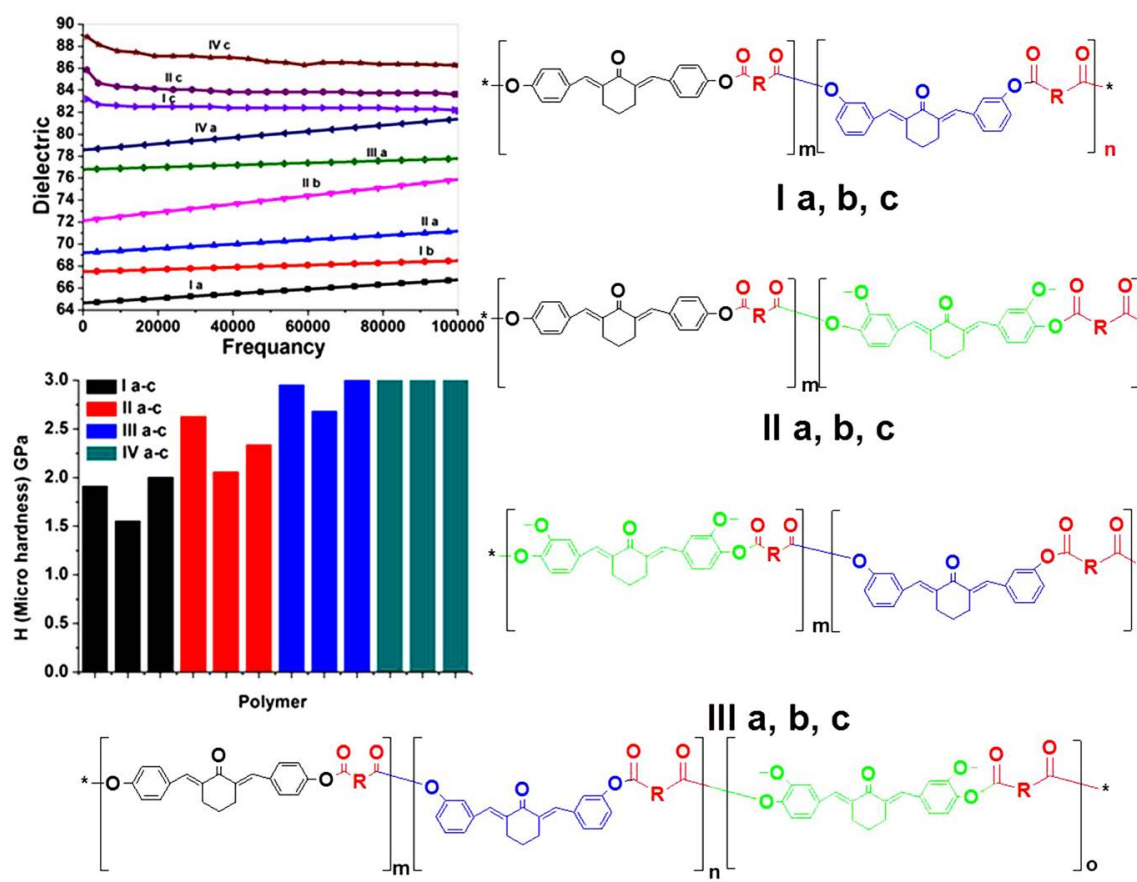

IV a, b, c 